
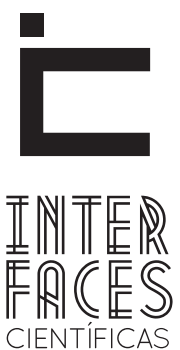

SAÚDE E AMBIENTE

\title{
PERFIL EPIDEMIOLÓGICO DOS PACIENTES ATENDIDOS PELA FISIOTERAPIA DO HOSPITAL DA UNIVERSIDADE FEDERAL DE SERGIPE
}

\author{
Lino Sérgio Rocha Conceição ${ }^{1}$ \\ Tássia Virgínia de Carvalho Oliveira ${ }^{3}$ \\ Juliana Dantas Andrade ${ }^{5}$ \\ Paulo Autran Leite Lima ${ }^{7}$
}

\author{
Gabriela Santos Andrade 2 \\ Lícia Santos Santana \\ Gabriela Silva Correia ${ }^{6}$
}

\section{RESUMO}

A análise do perfil de utilização de serviços de saúde em geral é complexa. As alterações do perfil demográfico e epidemiológico da população, as transformações na organização da assistência e a contínua incorporação e demanda por novas tecnologias implicam na necessidade de estudos cada vez mais detalhados da assistência hospitalar no país que apoiem a gestão do Sistema Único de Saúde (SUS) e do Sistema de Assistência Suplementar. Desta forma, o presente estudo visou traçar perfil epidemiológico dos pacientes atendidos pelo serviço de fisioterapia no hospital da Universidade Federal de Sergipe. Foi realizado um estudo epidemiológico descritivo dos atendimentos das enfermarias pelo serviço de fisioterapia do hospital universitário da Universidade Federal de Sergipe (HU-UFS). A amostra foi compreendida de 200 pacientes e pode-se ob- servar uma grande variedade de grupos de doenças notificadas, especialmente as de caráter oncológico e infectocontagioso. Foi evidente a grande quantidade de pacientes acometidos por doenças oncológicas, o que reforça a necessidade de maior atenção da equipe multiprofissional para o tratamento desses pacientes. Além disso, mais estudos são necessários para que se estabeleçam protocolos avançados de atendimento em vários grupos de doenças, especialmente os de carácter infectocontagiosa e parasitária, devido às complicações cinético-funcionais que essas doenças trazem para o indivíduo.

\section{PALAVRAS-CHAVE}

Epidemiologia. Perfil de Saúde. Fisioterapia. Serviço Hospitalar de Fisioterapia. 


\section{ABSTRACT}

A profile analysis of use of health services in general is complex. The changes in demographic and epidemiological profile of the population, changes in the organization of care and the continued development and demand for new technologies imply the need for increasingly detailed studies of hospital care in the country to support the management of the Unified Health System (SUS) and Supplemental Assistance System. Thus, the present study aims to outline epidemiological profile of patients treated by physiotherapy service at the Hospital of the Federal University of Sergipe. A descriptive epidemiological study of the care of the wards was conducted by physiotherapy service of the University Hospital of the Federal University of Sergipe (UFS$-\mathrm{HU})$. The sample was comprised of 200 patients, we can observe a wide variety of disease groups reported, especially oncology and infectious and contagious character. It was obvious the large number of patients suffering from malignancies, which reinforces the need for greater attention from the multidisciplinary team to treat these patients. In addition, more studies are needed in order to establish advanced care protocols in various disease groups, especially those of infectious and parasitic nature, due to functional kinetic complications that these diseases bring to the individual.

\section{KEYWORDS}

Epidemiology. Health Profile. Physicaltherapy. Physiotherapy Hospital Service.

\section{RESUMEN}

El análisis del perfil de la utilización de los servicios de salud, en general, es compleja. Los cambios en el perfil demográfico y epidemiológico de la población, y de la organización de la atención y el desarrollo continuo y la demanda de las nuevas tecnologías, implican la necesidad de estudios cada vez más detallados de la atención hospitalaria en el país, para apoyar a la gestión del Sistema Único de Salud (SUS) y el Sistema de Asistencia Suplementaria. Por lo tanto, el presente estudio tiene como objetivo delinear el perfil epidemiológico de los pacientes tratados por el servicio de fisioterapia en el Hospital de la Universidad Federal de Sergipe. Se realizó un estudio epidemiológico descriptivo de los asistidos por el departamento de fisioterapia del Hospital Universitario de la Universidad Federal de Sergipe (UFS-HU). La muestra estuvo compuesta por 200 pacientes, y se puede observar una gran variedad de grupos de enfermedades reportados, especialmente los relacionados con el cáncer e infectocontagioso. Ha quedado claro, el gran número de pacientes que sufren de enfermedades malignas, lo que refuerza la necesidad de una mayor atención por parte del equipo multidisciplinario para el tratamiento de estos pacientes. Además, se necesitan más estudios para establecer protocolos que la atención avanzada en varios grupos de enfermedades, sobre todo infecciosos y parasitarios, debido a las complicaciones funcionales cinéticas que estas enfermedades traen al individuo.

\section{PALABRAS CLAVE}

Epidemiología. Perfil de la Salud. Terapia Física. Fisioterapia atendida por el Hospital. 


\section{INTRODUCÃO}

O Sistema único de saúde (SUS) foi criado com intento de alterar a situação de desigualdade na assistência à saúde da população, tornando obrigatório o atendimento público a qualquer cidadão, sendo impedida a cobrança de valor sob qualquer pretexto. Esse sistema prevê uma rede regionalizada de ações e serviços, que oferece atenção integral à saúde da população. Em seus princípios organizativos, propõe a descentralização da coordenação, regionalização do atendimento e hierarquização dos serviços, defende a universalidade, a equidade, a integralidade e a participação social (YUNES, 1999; Sistema..., 2000; IBGE, 2008).

A análise do perfil epidemiológico de serviços de saúde em geral é complexa, ao resultar da articulação entre oferta e acesso aos serviços de saúde e das características sócio-demográficas e epidemiológicas dos usuários. As alterações do perfil demográfico e epidemiológico da população, as transformações na organização da assistência, a contínua incorporação e demanda por novas tecnologias implicam a necessidade de estudos cada vez mais detalhados da assistência hospitalar no país que apoiem a gestão do SUS e do Sistema de Assistência Suplementar (TRAVASSOS et al., 2000; TRAVASSOS; MARTINS, 2004).

Em termos práticos, o número de internações hospitalares por meio do SUS foi de 12.150 .040 entre o período de julho de 2011 a julho de 2012, sendo o sudeste e nordeste as regiões com os maiores indicadores, com 4.738 .733 e 3.376 .838 de internações, respectivamente (MINISTÉRIO DA SAÚDE, 2013).

A concepção tradicional define um hospital universitário (HU) como uma instituição caracterizada por ser um prolongamento de um estabelecimento de ensino em saúde que propicia atendimento médico de maior complexidade (nível terciário) a uma parcela da população. Entretanto, nas últimas duas décadas, ampliou-se nos países desenvolvidos a au- tonomia dos hospitais universitários, os quais passam a manter funções definidas nos sistemas de saúde, subordinando-se progressivamente à lógica dos serviços (MEDICl, 2001).

Na maioria dos hospitais de países desenvolvidos, a fisioterapia é vista como uma parte integrante do tratamento de seus pacientes. 0 papel preciso que este profissional desempenha varia consideravelmente de uma unidade para outra e depende de alguns fatores como financeiro e multidisciplinares. No leito, por exemplo, os pacientes podem sofrer pelos efeitos da imobilidade, que causam uma série de complicações. A capacidade de deambulação após o período de imobilidade pode ser prejudicada, dificultando consideravelmente as atividades de vida diária, ou, no pior dos casos, resultando em quedas ou piora da qualidade de vida. Além disso, imobilização prolongada pode resultar em danos, tais como úlceras de decúbito, pneumonia, e trombose.

Adicionalmente, a imobilização muitas vezes causa estresse e tem um impacto negativo sobre as habilidades cognitivas. Sendo assim, observa-se que a atuação fisioterapêutica é fundamental para o treinamento físico precoce no cuidado e recuperação do paciente, na melhora da capacidade funcional, autoavaliação do estado funcional e da força muscular na alta hospitalar (STILLER, 2000; WANG; MOYLE, 2005; GASTMANS; MILISEN, 2006; BURTIN et al., 2006).

A fisioterapia respiratória é frequentemente realizada na prevenção e tratamento de atelectasias pulmonares, pneumonias associadas e exacerbação de doenças obstrutivas pulmonares crônicas. Essa especialidade utiliza técnicas de treinamento muscular inspiratório em pacientes de alto risco para procedimentos cirúrgicos de uma forma geral e pode reduzir o risco de complicações pulmonares, uma vez que melhora a força e resistência dos músculos respira- 
tórios. Assim, devido o benefício obtido pela redução do impacto das complicações pulmonares, indica-se, a título de exemplo, a fisioterapia intensiva, que tende a aumentar a saturação de oxigênio, reduzir tempo de internação hospitalar, e alterar a distribuição da ventilação/perfusão. Ela tem um efeito positivo significativo sobre a capacidade de exercício dos pacientes com neoplasia pulmonar (PEHLIVAN et al., 2011).

Adicionalmente, indivíduos instruídos e orientados a exercícios ventilatórios fisioterapêuticos e rotinas hospitalares no período pré-operatório apresentaram níveis de ansiedade menores quando comparados aos indivíduos que não receberam orientação. Foi correlacionado que pacientes mais ansiosos apresentam, no pós-cirúrgico, maiores níveis de dor e permanência no hospital (GARBOSSA et al., 2009).

A fisioterapia precoce, ainda no período hospitalar, por meio de diferentes técnicas cinesioterapêuticas, é eficaz em todas as fases da doença, previne deformidades, proporciona maior independência funcional e melhora a qualidade de vida. O exercício físico promove efeitos fisiológicos benéficos com o aumento do fluxo sanguíneo, a melhor distribuição do oxigênio na interface célula-capilar e gera mudanças significativas no sistema cardiovascular pelo aprimoramento do sistema de transporte, da extração e da utilização do oxigênio, enquanto que no sistema respiratório melhora a função pulmonar com o aumento do volume corrente, da capacidade pulmonar e da capacidade de difusão. Benefícios psicológicos, também, ocorrem e promovem sensação de bem-estar, melhoram a autoestima e a qualidade de vida (CAVENAGHI, 2005).

Em um estudo analítico, foi comparado o tratamento eletroterapêutico e cinesioterapêutico na reabilitação de 19 pacientes com lesão medular, observou-se que ambos os métodos mostraram redução de edemas, de úlceras de pressão, de bloqueio articular e do tônus muscular desses indivíduos. Para o tratamento cinesioterapêutico foram aplicados exercícios de fortalecimento muscular, de manutenção da am- plitude de movimento, facilitação de movimentos ativos na musculatura disponível, alongamentos e transferências (PEIXOTO et al., 2003).

Apesar da importância da fisioterapia no ambiente hospitalar, não há trabalhos que avaliem o perfil epidemiológico em hospitais universitários, justificando essa pesquisa. 0 reconhecimento do perfil epidemiológico representa uma demanda importante para que se obtenha uma melhor adequação das práticas de saúde, bem como estabelecer uma demanda ambulatorial na rede pública para uma avaliação fidedigna dos serviços, com o objetivo de orientar todos aqueles envolvidos na gestão e planejamento em saúde. Nesse contexto, o presente estudo visou traçar o perfil epidemiológico dos pacientes atendidos pelo serviço de fisioterapia no hospital universitário da Universidade Federal de Sergipe.

\section{METODOLOGIA}

Foi realizado um estudo epidemiológico descritivo dos atendimentos das enfermarias pelo serviço de fisioterapia do hospital universitário da Universidade Federal de Sergipe (HU-UFS). Foram coletados dados por meio de registros de estatística dos pacientes atendidos no período de 1/6/2011 a 31/5/2012 e inclusos todos pacientes do SUS que tiveram prescrição de tratamento fisioterapêutico no hospital. Não foram coletados dados referentes a nome, naturalidade, local de residência, profissão, estado civil e raça/cor. Foram excluídos dados de paciente sem diagnóstico clínico, com diagnóstico clínico inconclusivo, com grafia ilegível e a amostra foi selecionada por conveniência.

O banco de dados da pesquisa foi compreendido por diagnóstico clínico, setor de internação, idade e gênero. Depois de realizado o levantamento de dados, estes foram digitados em um programa para edição de texto, em seguida os dados obtidos foram lançados no programa microsoft excel 2010, para elaboração e formatação de planilhas e gráficos. Nesse trabalho foi utilizada estatística simples com média e desvio padrão. 


\section{RESULTADOS}

A amostra da pesquisa foi compreendida de 200 pacientes atendidos pelo serviço de fisioterapia do HU-UFS, sendo 101 do gênero masculino $(51,5 \%)$ e 99 do gênero feminino $(48,5 \%)$ e a média de idade foi de $52,6 \pm 18,7$ anos.

Outro aspecto observado nessa análise foi que as especialidades clínicas mais prevalentes foram às doenças oncológicas, totalizando 44 pacientes (22\%) distribuídos em todos os setores de atendimento. Em um segundo plano, encontraram-se as de ordem infectocontagiosas, totalizando 41 pacientes (20\%). Doenças respiratórias e outras com etiologias desconhecidas ou relacionadas ao envelhecimento demonstraram valores expressivos, compreendendo $10 \%$ para os dois grupos.

Ainda dentro da análise gráfica do perfil epidemiológico por especialidade, observou-se uma similaridade na prevalência de doenças do trato digestivo, doenças parasitárias, reumato-ortopédicas e doenças neurológicas. Estas totalizaram junto um percentual de $24 \%$. Os demais valores compreenderam: procedimentos cirúrgicos (5\%), seguido pelas doenças cardiovasculares (4\%), doenças uroginecológicas (4\%), e doenças endócrinas (2\%). Esses dados podem ser observados no Gráfico 1.

\section{Gráfico 1 - Perfil Epidemiológico por Especialidade}

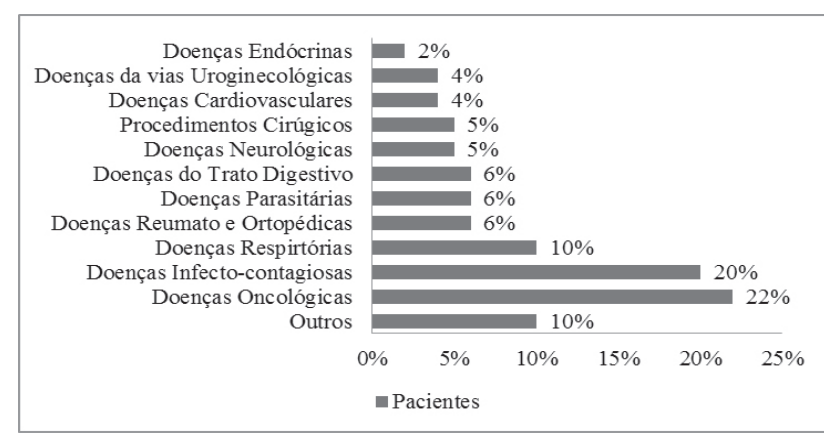

Fonte: Dados da pesquisa.
Dentro da logística de locação de pacientes por setores, o HU-UFS está organizado em três centros de atendimento, clinica médica I, clínica médica II, e clínica cirúrgica I. Nesse aspecto, pode-se observar no Gráfico 2, que o setor que apresentou a maior demanda de atendimentos foi a clínica médica II com um percentual de $47 \%$ dos atendimentos, seguido pela clínica cirúrgica I com $33 \%$ dos atendimentos e, por fim, a clínica médica I corresponde a $20 \%$ das demandas de atendimento.

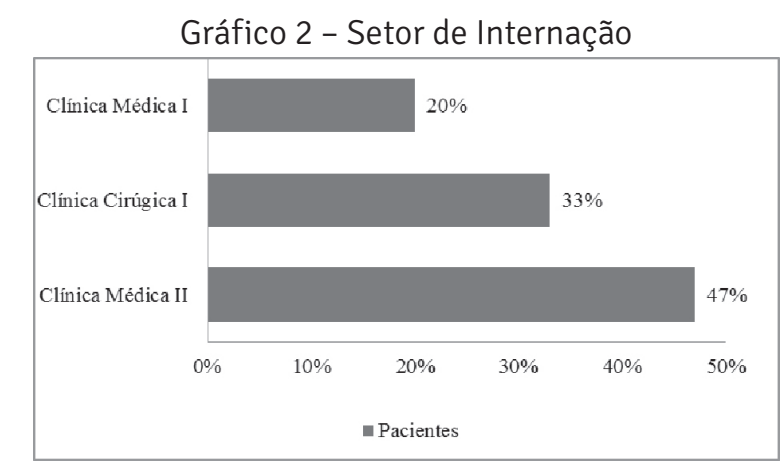

Fonte: Dados da pesquisa.

Em uma análise adicional (Gráfico 3) foi observado a proporção do gênero masculino e feminino nas diferentes áreas de atendimento da fisioterapia HU-UFS. Em uma observação geral, foi possível observar que existe uma predominância do gênero masculino, por área da saúde, em comparação ao gênero feminino.

Gráfico 3 - Proporção patológica no gênero masculino e feminino

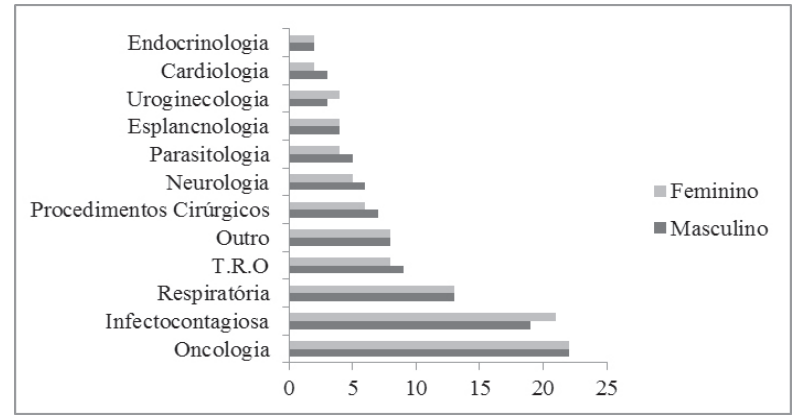

Legenda: Dados expressos em porcentagem. T.R.O - Traumatologia, reumatologia e ortopedia. Fonte: Dados da pesquisa. 
No Gráfico 4 pode ser observado um grupo específico, o grupo das doenças infecto contagiosas e parasitárias, que correspondem juntas a $26 \%$ das patologias atendidas pela fisioterapia do HU-UFS. Nele é possível observar a grande prevalência da SIDA/HIV, correspondendo a 38\% dos pacientes atendidos nessa especialidade. Em seguida, patologias que ainda apresentam valores expressivos, a bronquiectasia com $27 \%$, a leishmaniose visceral, com $13 \%$ e pneumonias com $8 \%$.

Gráfico 4 - Prevalência entre as doenças infectocontagiosas e parasitárias

\section{Prevelência de lesão no TGI}

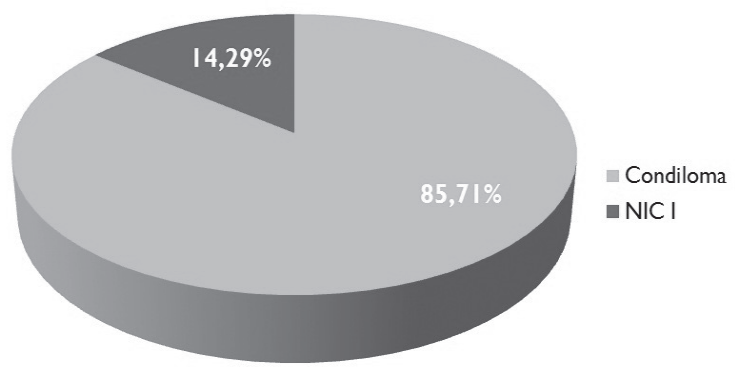

Fonte: Dados da pesquisa.

\section{DISCUSSÃO}

Em uma análise inicial do estudo foi observado um número maior de indivíduos do gênero masculino. Dados do DATASUS (2008) apontaram que na região nordeste houve uma prevalência de relatos de internações hospitalares pelo gênero feminino, com 54,1\%, em comparação ao gênero masculino, que apresentou uma proporção de 45,9\%. Apesar da falta de dados atualizados, pode-se observar em neste estudo um percentual de $51,5 \%$ para o gênero masculino, e $48,5 \%$ para o gênero feminino, evidenciando uma distribuição equiparada entre gêneros.
A média de idade, 52,6 $\pm 18,7$ anos, é justificada pelo perfil de atendimento do hospital, que engloba a atenção à saúde do adulto e do idoso. Em outro levantamento do DATASUS (2013) referente à morbidade hospitalar, observou-se que nas faixas etárias que englobam a saúde do adulto e do idoso, no período de julho de 2012, foram de 713.732 internações em todo o Brasil, sendo que a região nordeste apresentou o terceiro maior valor, com 186.847, englobando a faixa etária em questão.

Em relação à alocação dos pacientes, observa-se que a grande maioria dos pacientes foi atendida no setor das clínicas médicas I e II, que juntas compreenderam o valor de $67 \%$ dos leitos ocupados, em comparação aos pacientes internados para cirurgias eletivas no setor da clínica cirúrgica I correspondendo a $33 \%$ dos leitos ocupados. Essa característica do atendimento se apresenta possivelmente em virtude da grande maioria dos pacientes não necessitarem de procedimentos cirúrgicos ou não estarem aptos para os mesmos. Apesar das doenças neoplásicas apresentarem a maior incidência e um dos métodos de tratamento eficaz ser o procedimento cirúrgico, os valores não refletem essa demanda dentro do presente trabalho.

Segundo dados do Instituto Nacional do Câncer (INCA, 2013) referentes à distribuição de taxas de incidência das neoplasias malignas, em Aracaju foram registrados cerca de 213,9 e 292,7 casos (por 100.000 habitantes), no gênero feminino e masculino respectivamente. Além disso, em 2012, no estado de Sergipe, foram estipulados 163,7 casos para cada 100 mil homens e 165,1 casos para cada 100 mil mulheres. Os dados do instituto não englobam os valores com respeito às neoplasias tegumentares. Em comparação ao presente estudo no qual as doenças oncológicas totalizaram 44 (22\%) pacientes atendidos pelo serviço. Não houve diferença na relação entre gêneros, pois o número de pacientes para o grupo das neoplasias malignas foi de 22 pacientes do gênero masculino e 22 do gênero feminino. 
Muitos pacientes com câncer sofrem prejuízos na vida cotidiana, como resultado de efeitos adversos ou sequelas associadas ao tratamento, com o desenvolvimento, e a fase terminal da sua doença. A reabilitação pode desempenhar um papel importante como uma abordagem para a manutenção e melhoria da qualidade de vida de pacientes com câncer. A reabilitação oncológica foi classificada de acordo com as necessidades físicas e individuais dos pacientes oncológicos em quatro categorias: preventiva, restaurativa, de suporte e paliativa (DIETZ, 1969).

No que diz respeito ao tratamento das doenças oncológicas, espera-se que a reabilitação seja uma ferramenta na manutenção ou melhora das atividades de vida diária desses pacientes, associado ao suporte, objetivando manter a qualidade de vida de pacientes e familiares (OKAMURA, 2011). Em outro estudo, concluiu-se que a atividade física tem efeitos positivos sobre a fisiologia, composição corporal, das funções físicas, os resultados psicológicos e qualidade de vida em pacientes após o tratamento para câncer de mama. Quanto aos pacientes com outro tipo de câncer, a atividade física foi associada com a redução do IMC e peso corporal e melhoria da qualidade de vida (FONG et al., 2012).

O HU-UFS é um centro de saúde de média complexidade que possui um enfoque no atendimento as doenças infectocontagiosas. Esta por sua vez, representa o segundo grupo de doenças com maior prevalência de atendimento pelo serviço de fisioterapia, compreendendo $20 \%$ dos pacientes. Observando o Gráfico 4 constatou-se que, dentre o referido grupo, a doença com maior prevalência foi a síndrome da imunodeficiência adquirida (SIDA/AIDS) compreendendo 38\% dos pacientes.

A fisioterapia, enquanto ciência voltada à reabilitação e promoção à saúde do indivíduo SIDA/HIV tem um campo vasto de atuação. Entretanto, é necessário compreender todo o processo infeccioso do vírus HIV, sua sintomatologia, além dos efeitos adversos e do uso contínuo de terapia antirretroviral. 0 tratamento fisioterapêutico para portadores de SIDA/HIV visa propor- cionar atividades educativas e terapêuticas voltadas à prevenção das alterações funcionais dos seus portadores, incrementando a qualidade de vida física, psíquica e social. No âmbito clínico/funcional, o profissional de fisioterapia, inserido em uma equipe multidisciplinar de saúde (públicos/privados) em SIDA/HIV, pode prescrever condutas terapêuticas de acordo com as necessidades objetivas e subjetivas de cada paciente (NOBRE, 2008).

0 contexto da fisioterapia dentro de campos como a traumatologia, reumatologia, ortopedia, pneumologia e cardiologia apresenta boa documentação cientifica no que diz respeito a sua atuação nessas respectivas áreas (WILK et al., 2012; KETEYIAN et al., 2012; MERELES et al., 2006). Adicionalmente, a atuação da fisioterapia nas doenças metabólicas e endócrinas se mostra promissora. Em um estudo randomizado foi demonstrado que tanto a marcha como o equilíbrio de pacientes com diabetes mellitus pode ser melhorada. Foi evidenciado que o treinamento de marcha associado ao treinamento de equilíbrio com base em uma abordagem de circuitos, pode melhorar a velocidade de marcha e equilíbrio, aumentar a força muscular e mobilidade articular de pacientes diabéticos (ALLET et al., 2010).

Em outro âmbito estão as doenças parasitárias, entre as quais a mais prevalente no setor de atendimento da fisioterapia foi à leishmaniose visceral (LV), com o percentual de $13 \%$. A LV é uma infecção parasitária que atinge tanto adultos quanto crianças, apresentando-se tipicamente com quadro de febre, hepatoesplenomegalia e pancitopenia. A doença é marcada por diversas alterações hematológicas, bioquímicas e imunológicas como: anemia, leucopenia acentuada, plaquetopenia, alterações das enzimas hepáticas e proteínas plasmáticas, albumina baixa e globulinas elevadas, dentre outros. Um estudo avaliou o surgimento de pneumonia em pacientes com diagnóstico clínico de LV e comparou a incidência de pneumonia e tempo de internação. Pode se comprovar estatisticamente que esses pacientes puderam se beneficiar com a fisioterapia respiratória na prevenção de pneumonia associada (ÁVILA et al., 2008). 
A hanseníase é causada por Mycobacterium leprae e manifesta-se como lesões cutâneas e nervos periféricos. Sabe-se que o bacilo é considerado de baixa patogenicidade, pois apenas uma pequena parcela dos indivíduos supostamente infectados desenvolve a moléstia. A doença por muitos anos tem sido foco na saúde do Brasil. Em 2006 a Hanseníase foi incluída no pacto pela saúde do SUS. Esse pacto reforça no SUS o movimento da gestão pública por resultados, estabelece um conjunto de compromissos sanitários considerados prioritários, pactuado de forma tripartite, a ser implementado pelos entes federados. Esses compromissos deverão ser efetivados pela rede do SUS, de forma a garantir o alcance das metas pactuadas. Possui algumas prioridades dentre as quais está o fortalecimento da capacidade de respostas às doenças emergentes e endemias, com ênfase, além de outras doenças, na hanseníase. Pode-se observar nos dados do Gráfico 4, apesar de ser um dado pouco expressivo diante das outras doenças.

A eliminação da hanseníase tem sido difícil de ser alcançada em algumas regiões, em face da complexidade da doença. Diante do não conhecimento de todos os aspectos clínicos e laboratoriais da hanseníase, como a inexistência de um modelo experimental sólido e a impossibilidade de se cultivar o $\mathrm{M}$. leprae, torna-se difícil a compreensão de pontos cruciais da enfermidade, como a transmissão, o neurotropismo da micobactéria e a suscetibilidade de alguns indivíduos para o desenvolvimento de formas mais graves ou mais baciliferas (DUARTE-CUNHA et al., 2012). A atuação fisioterapêutica é de grande importância no que diz respeito à prevenção de incapacidades, devido ao acometimento de nervos periféricos, e também as abordagens fisioterapêuticas nas plantares decorrentes da hanseníase (DUARTE-CUNHA, 2012).

Pouco se conhece a respeito da conduta fisioterapêutica nas repercussões de doenças como aspergiloma, leptospirose, erisipela e doença de chagas no organismo. A doença de chagas, por exemplo, apresenta bons resultados diante da reabilitação. Em um estudo, quatorze mulheres portadoras de doença de Chagas na faixa etária entre 40 e 60 anos, classe I da New York He- art Association (NYHA) foram divididas em dois grupos. Sete no G1 (Grupo 1) que realizaram exercício físico, com duas sessões diárias semanais por seis semanas e sete no G2 (Grupo 2) sem tal intervenção. Observaram-se na avaliação final, diferenças significativas na pressão arterial sistólica, no pico do fluxo expiratório, nas pressões inspiratória e expiratória máximas e na frequência cardíaca. Houve aumento significativo na ergoespirometria, no MET (equivalente metabólico) máximo, no estágio do protocolo de Bruce, no Vo (consumo máximo de oxigênio) máximo, na duração do teste e na distância percorrida para as participantes de G1 e apenas na duração do teste para G2 (MENDES et al., 2011).

\section{CONCLUSÃO}

Por meio deste estudo foi possível traçar o perfil epidemiológico dos pacientes atendidos pelo serviço de fisioterapia do HU-UFS através da análise da demanda de pacientes e dos grupos de doenças apresentados. Pode-se evidenciar que o fisioterapeuta é um membro importante da equipe multiprofissional, atuando de forma generalista.

Foi evidente a grande quantidade de pacientes acometidos por doenças oncológicas, o que reforça a necessidade de maior atenção da equipe multiprofissional para o tratamento desses pacientes.

Apesar disso, mais estudos são necessários para que se estabeleçam protocolos avançados de atendimento em vários grupos de doenças, especialmente as de caráter infectocontagioso e parasitário, devido às complicações cinético-funcionais que essas doenças trazem para o indivíduo, e por algumas delas serem endêmicas na região nordeste do Brasil. Desta forma, uma caracterização epidemiológica para um melhor planejamento, gestão e evolução se faz necessária dentro da saúde pública no Brasil. Como sugestão para trabalhos futuros, um espaço temporal de maior abrangência e estratificação dos grupos de doenças de risco dentro do atendimento ou outras doenças de ordem endêmica que são subnotificadas para o tratamento fisioterapêutico. 


\section{REFERÊNCIAS}

ALLET, L. et al. The gait and balance of patients with diabetes can be improved: a randomised controlled trial. Diabetologia, v.53, n. 3, p.458-66, 2010.

ÁVILA P. E. S. A fisioterapia respiratória na prevenção de pneumonia em pacientes com leishmaniose visceral (calazar). Rev Para Med, v.22, n.4, 2008.

BURTIN, C. et al. Effectiveness of early exercise in critically ill patients: preliminary results. Intensive Care Med, v.32, p.109, 2006.

CAVENAGHI, S. et al. Aplicabilidade intra-hospitalar da cinesioterapia no trauma raquimedular. Arq Ciênc Saúde, v.12, n.4, p.213-15, 2005.

DATASUS /IBGE - Pesquisa Nacional por Amostra de Domicílios - PNAD - Suplemento Saúde, 2008. Disponível em <http://tabnet.datasus.gov.br/cgi/ dh.exe?idb2011/f24.def>. Acesso em: 19 abr. 2014.

DATASUS /IBGE - Pesquisa Nacional por Amostra de Domicílios - PNAD - Suplemento Saúde, 2013. Disponível em <http://tabnet.datasus.gov.br/cgi/ dh.exe?idb2011/f24.def >. Acesso em: 19 abr. 2014

DIETZ, J. H. JR. Rehabilitation of the cancer patients. Med Clin North Am, v.53, p.607-624, 1969.

DUARTE-CUNHA, M. Aspectos epidemiológicos da hanseníase: uma abordagem espacial. Cad. Saúde Pública, v.28, n.6, p.1143-1155, 2012.

DUARTE-CUNHA, M. et al. Aspectos epidemiológicos da hanseníase: uma abordagem espacial. Cad. Saúde Pública [online], v.28, p.1143-1155, 2012.

FONG, D. Y. Physical activity for cancer survivors: meta-analysis of randomised controlled trials. British Medical Journal, v.344, n.70, p.1-14, 2012.
GARBOSSA, A. et al. Effects of physiotherapeutic instructions on anxiety of CABG patients. Rev Bras Cir Cardiovasc, v.24, n.3, p.359-366, 2009.

GASTMANS, C.; MILISEN K. Use of physical restraint in nursing homes: clinical ethical considerations. J Med Ethics, v.32, n.3, p.148-52, 2006.

IBGE - Pesquisa Nacional por Amostra de Domicílios - PNAD - Suplemento Saúde, 2008.

Instituto Nacional do Câncer (INCA), 2013. Disponível em: <http://www.inca.gov.br/cancernobrasil/2010/docs/taxa_neoplasias/Parte451_registro_ de_base_populacional_completo.pdf $>$. Acesso em: 10 abr. 2013.

KETEYIAN, S. J. Relation between volume of exercise and clinical outcomes in patients with heart failure. J Am Coll Cardiol, v.60, n.19, p.1899-905, 2012.

MEDICI, A. C. Hospitais Universitários: passado, presente e futuro. Assoc Med Bras, v.47, n.2, p.149-56, 2001.

MENDES, M. F. A. Exercício físico aeróbico em mulheres com doença de Chagas. Fisioter. Mov., v.24, p 591601, 2011.

MERELES, D. et al. Exercise and Respiratory Training Improve Exercise Capacity and Quality of Life in Patients With Severe Chronic Pulmonary Hypertension. Circulation, v.114, n. 4, p.1482-1489, 2006.

MINISTÉRIO DA SAÚDE - Sistema de Informações Hospitalares do SUS-SIH/SUS, 2013. Disponível em: <http://portal.saude.gov.br/portal/saude/profissional/area.cfm?id_area $=1338>$. Acesso em: 13 abr. 2014. 
NOBRE, A. Q. T. et al. A fisioterapia no contexto do HIV/AIDS. Fisioter. Mov, v.21, n.4, p.11-18, 2008.

OKAMURA, H. Importance of rehabilitation in cancer treatment and palliative medicine. Jpn J Clin Oncol, v.41, n.6, p.733-8, 2011.

PEHLIVAN E, et al. The effects of preoperative short-term intense physical therapy in lung cancer patients: a randomized controlled trial. Ann Thorac Cardiovasc Surg, v.17, n.5, p.461-8, 2011.

PEIXOTO, B. O. et al. Lesão medular: estudo do potencial evocado como recurso prognóstico e comparação entre o tratamento de estimulação elétrica neuromuscular e a fisioterapia convencional. Fisioter Bras, v.4, n.1, p.17-23, 2003.

SISTEMA ÚNICO DE SAÚDE (SUS): Princípios e conquistas/ Ministério da Saúde, Secretaria ExecutivaBrasília: Ministério da Saúde, 2000.

STILLER, K. Physiotherapy in Intensive Care: Towars an evidence - bases practice. Chest, v.118, n.6, p.18011813, 2000.
TRAVASSOS, C. et al. Desigualdades geográficas e sociais na utilização de serviços de saúde no Brasil. Ciência \& Saúde Coletiva, v.5, n.1, p.133-149, 2000.

TRAVASSOS, C.; MARTINS, M. Uma revisão sobre os conceitos de acesso e utilização de serviços de saúde. Cad. Saúde Públ., Rio de Janeiro, v.20, supl. esp., p.190-198, 2004.

WANG, W. W.; MOYLE, W. Physical restraint use on people with dementia: a review of the literature. Aust J Adv Nurs, v.22, n.4, p.46-52, 2005

WILK, K. E. Recent advances in the rehabilitation of anterior cruciate ligament injuries. Orthop Sports Phys Ther, v.42, n.3, p.153-71, 2012.

YUNES, J. O SUS na lógica da descentralização. Estudos avançados, v.13, n.35, p.65-70, jan./abr. 1999.

1. Fisioterapeuta residente no Hospital Universitário da Universidade Federal de Sergipe. E-mail: linosergiorocha@gmail.com

2. Discente de Fisioterapia pela Universidade Tiradentes. E-mail: gab_sandrade@hotmail.com

3. Professora assistente da Universidade Tiradentes, mestre em Saúde e Ambiente pela Universidade Tiradentes. Universidade Tiradentes. E-mail: tassinhafisio@yahoo.com.br

4. Professora assistente da Universidade Tiradentes, mestre em Ginecologia e Obstetrícia da FMRP/USP e doutoranda em Ginecologia e Obstetrícia da FMRP/USP. Universidade Tiradentes. E-mail: licia2S@hotmail.com

5. Fisioterapeuta no Hospital Universitário da Universidade Federal de Sergipe, Hospital Universitário. E-mail: judantasandrade@yahoo.com.br

6. Fisioterapeuta no Hospital Universitário da Universidade Federal de Sergipe, Hospital Universitário. E-mail: gabcorreia@hotmail.com

7. Professor titular da Universidade Tiradentes, pós-doutor em Ciências das Saúde. Universidade Tiradentes. E-mail: pauloautranlima@gmail.com 